\title{
OPEN Clinical features and KRAS mutation in colorectal cancer with bone metastasis
}

\author{
Hyung Soon Park ${ }^{1,2}$, You Jin Chun ${ }^{3}$, Han Sang Kim ${ }^{3}$, Jee Hung Kim4, Choong-kun Lee ${ }^{3}$, \\ Seung-Hoon Beom ${ }^{3}$, Sang Joon $\mathrm{Shin}^{3}$ \& Joong Bae Ahn ${ }^{3 凶}$
}

Bone metastasis is known as a poor prognostic factor in colorectal cancer (CRC), but its clinical manifestations and outcomes are uncertain. CRC with bone metastasis was searched from January 2006 to April 2016. Of 11,551 CRC patients, 321 (2.7\%) patients had bone metastasis. Bone-only metastasis was found in only $8.7 \%$ of patients. Synchronous bone metastasis was present in 147 (45.8\%) patients. In patients with metachronous bone metastasis, the median time from CRC diagnosis to bone metastasis (TTB) was $\mathbf{2 7 . 2}$ months. KRAS mutation status was a marginally significant factor affecting TTB (median TTB, KRAS wild-type or mutation: 29 or 25.8 months, respectively, $\mathrm{P}=0.068$ ). Skeletal-related events (SREs) were noted in 200 (62.3\%) patients. Median overall survival (OS) from diagnosis of bone metastasis was 8.0 months. On multivariate analysis, multi-organ metastasis, peritoneal metastasis, neutrophil-to-lymphocyte ratio (NLR) $\geq 2.7$, and alkaline phosphatase (ALP) $\geq 123$ were independent factors for OS. Palliative chemotherapy prolonged survival in CRC patients with bone metastasis (HR $0.25,95 \% \mathrm{Cl} 0.2-0.33$ ). In conclusion, bone metastasis of CRC is rare, but it is related to SREs. Most patients have other organ metastasis and survival is $\mathbf{8 . 0}$ months. Attention should be paid to bone metastasis in CRC patients.

Colorectal cancer (CRC) was the fourth most common cancer in the United States, with a total of 101,420 cases of newly diagnosed CRC in $2019^{1}$. The most common metastatic sites were liver (70\%) and lung $(37 \%)^{2}$. Bone metastasis was observed in $1.2-12 \%$ of patients with CRC. The ten-year incidence of bone metastasis from CRC has been reported to be $2.7 \%^{3,4}$. Recently, bone metastasis incidence has increased due to improvement of survival of patients with metastatic CRC and diagnostic imaging techniques ${ }^{5}$. Bone metastasis of CRC is related to poor survival outcome. The median survival of patients with bone metastasis of CRC ranges from 7 months to 9.4 months $^{6-8}$. Previous studies have reported that multiple bone metastases, lung metastasis, and elevated carcinoembryonic antigen (CEA) are poor prognostic factors in CRC patients. However, these factors have not been universally observed in previous reports. The prognostic factors of patients with bone metastasis are still uncertain. A prognostic model has not been suggested yet. In addition, the role of palliative chemotherapy in CRC patients with bone metastasis has been rarely studied.

KRAS mutations have been detected in $40-50 \%$ of patients with CRC $^{9,10}$. KRAS mutations at codon 12 or 13 account for approximately $90 \%$ of all mutation types. In general, clinical behaviors are more aggressively presented in patients with KRAS mutations. Recurrence-free survival is significantly worse and lung metastasis is significantly higher in CRC patients with KRAS mutations ${ }^{11-13}$. Although the prognostic value of KRAS mutations is still controversial, some studies have revealed that KRAS mutations are associated with poorer survival ${ }^{12,14-16}$. However, associations between KRAS mutations and bone metastases have not been reported yet.

Thus, the aim of this study was to explore the clinical features of CRC patients with bone metastasis, including time to bone metastasis (TTB), metastatic sites of bone, and skeletal-related events (SREs) by KRAS mutation status. In addition, prognostic factors for CRC with bone metastasis were evaluated, and a prognostic model for survival was also suggested.

\footnotetext{
${ }^{1}$ Department of Internal Medicine, College of Medicine, The Catholic University of Korea, Seoul, Korea. ${ }^{2}$ Division of Medical Oncology, Department of Internal Medicine, St. Vincent's Hospital, The Catholic University of Korea, Suwon, Korea. ${ }^{3}$ Division of Medical Oncology, Department of Internal Medicine, Yonsei University College of Medicine, Seoul, Korea. ${ }^{4}$ Division of Medical Oncology, Department of Internal Medicine, Gangnam Severance Hospital, Yonsei University College of Medicine, Seoul, Korea. ${ }^{\circledR}$ email: vvswm513@yuhs.ac
} 


\begin{tabular}{|c|c|c|c|c|c|c|c|c|}
\hline & & \multirow{2}{*}{\multicolumn{2}{|c|}{ All patients }} & \multicolumn{5}{|l|}{ KRAS } \\
\hline & & & & \multicolumn{2}{|l|}{ Wild type } & \multicolumn{2}{|c|}{ Mutant type } & \multirow[b]{2}{*}{ P-value } \\
\hline & & No. (321) & $\%$ & No. (126) & $\%$ & No. $(78)$ & $\%$ & \\
\hline Age & (Median, range) & 61 & $28-87$ & 58 & $29-83$ & 62 & $28-79$ & 0.007 \\
\hline \multirow{2}{*}{ Sex } & Male & 188 & 58.6 & 81 & 64.3 & 38 & 48.7 & 0.028 \\
\hline & Female & 133 & 41.4 & 45 & 35.7 & 40 & 51.3 & \\
\hline \multirow{2}{*}{ Primary site } & Colon & 192 & 59.8 & 79 & 62.7 & 48 & 61.5 & 0.868 \\
\hline & Rectum & 129 & 40.2 & 47 & 37.3 & 30 & 38.5 & \\
\hline \multirow{2}{*}{ Tumor sideness } & Left side & 250 & 77.9 & 105 & 84.7 & 55 & 71.4 & 0.023 \\
\hline & \begin{tabular}{|l|} 
Right side \\
\end{tabular} & 63 & 19.6 & 19 & 15.3 & 22 & 28.6 & \\
\hline \multirow{2}{*}{ Metastasis pattern } & Synchronous & 147 & 45.8 & 55 & 43.7 & 31 & 39.7 & 0.583 \\
\hline & Metachronous & 174 & 54.2 & 71 & 56.3 & 47 & 60.3 & \\
\hline \multirow{3}{*}{ Histologic type } & Adenocarcinoma & 292 & 91 & 114 & 90.5 & 73 & 93.6 & 0.325 \\
\hline & SRC & 14 & 4.4 & 7 & 5.6 & 1 & 1.3 & \\
\hline & Mucinous carcinoma & 15 & 4.7 & 5 & 4 & 4 & 5.1 & \\
\hline \multirow{3}{*}{ Clinical stage at CRC diagnosis } & 1 or 2 & 19 & 5.9 & 7 & 5.7 & 6 & 7.7 & 0.283 \\
\hline & 3 & 68 & 21.2 & 32 & 26 & 13 & 16.7 & \\
\hline & 4 & 229 & 71.3 & 84 & 68.3 & 59 & 75.6 & \\
\hline \multirow{2}{*}{ Bone only metastasis } & Yes & 28 & 8.7 & 9 & 7.1 & 2 & 2.6 & 0.211 \\
\hline & No & 293 & 91.3 & 117 & 92.9 & 76 & 97.4 & \\
\hline \multicolumn{9}{|l|}{\begin{tabular}{|l|} 
Other organ metastasis \\
\end{tabular}} \\
\hline \multirow{2}{*}{ Liver metastasis } & No & 137 & 42.7 & 59 & 46.8 & 22 & 28.2 & 0.008 \\
\hline & Yes & 184 & 57.3 & 67 & 53.2 & 56 & 71.8 & \\
\hline \multirow{2}{*}{ Lung metastasis } & No & 153 & 47.7 & 63 & 50 & 28 & 35.9 & 0.049 \\
\hline & Yes & 168 & 52.3 & 63 & 50 & 50 & 64.1 & \\
\hline \multirow{2}{*}{ Peritoneal metastasis } & No & 243 & 75.7 & 90 & 71.4 & 60 & 76.9 & 0.387 \\
\hline & Yes & 78 & 24.3 & 36 & 28.6 & 18 & 23.1 & \\
\hline \multirow{2}{*}{ Brain metastasis } & No & 306 & 95.3 & 122 & 96.8 & 70 & 89.7 & 0.062 \\
\hline & Yes & 15 & 4.7 & 4 & 3.2 & 8 & 10.3 & \\
\hline \multicolumn{9}{|l|}{ Site of bone metastasis } \\
\hline \multirow{2}{*}{ Spine } & No & 100 & 31.2 & 37 & 29.4 & 29 & 37.2 & 0.246 \\
\hline & Yes & 221 & 68.8 & 89 & 70.6 & 49 & 62.8 & \\
\hline \multirow{2}{*}{ Pelvis } & No & 155 & 48.3 & 60 & 47.6 & 39 & 50 & \\
\hline & Yes & 166 & 51.7 & 66 & 52.4 & 39 & 50 & \\
\hline \multirow{2}{*}{ Long bone } & No & 252 & 78.5 & 100 & 79.4 & 62 & 79.5 & 0.983 \\
\hline & Yes & 69 & 21.5 & 26 & 20.6 & 16 & 20.5 & \\
\hline \multirow{2}{*}{ Other bone $\mathrm{a}^{\mathrm{a}}$} & No & 197 & 61.4 & 79 & 62.7 & 50 & 64.1 & 0.84 \\
\hline & Yes & 124 & 38.6 & 47 & 37.3 & 28 & 35.9 & \\
\hline & NLR & 3.5 & $0.4-31.8$ & 3.5 & $0.6-19.7$ & 3.6 & $0.8-31.8$ & 0.937 \\
\hline Laboratory values at diagnosis of bone metastasis (median, & Platelet $(\times 1000 / \mathrm{uL})$ & 247 & $25-654$ & 243 & $26-637$ & 245 & $25-654$ & 0.903 \\
\hline range) & ALP (IU/L) & 114 & $17-1509$ & 98 & $17-897$ & 136 & $32-1509$ & 0.025 \\
\hline & CEA (ng/mL) & 75.4 & $0.4-20,000$ & 77.7 & $0.4-20,000$ & 159.3 & $2.3-20,000$ & 0.041 \\
\hline & Irinotecan based & 96 & 29.9 & 47 & 37.3 & 32 & 41 & 0.136 \\
\hline Pallintive CT & Oxaliplatin based & 73 & 22.7 & 33 & 26.2 & 10 & \begin{tabular}{|l|}
12.8 \\
\end{tabular} & \\
\hline Palliative CIX & Others $^{\mathrm{b}}$ & 43 & $\mid 13.4$ & 16 & \begin{tabular}{|l|}
12.7 \\
\end{tabular} & 11 & \begin{tabular}{|l|}
14.1 \\
\end{tabular} & \\
\hline & No & 109 & 34 & 30 & 23.8 & 25 & 32.1 & \\
\hline
\end{tabular}

Table 1. Characteristics of CRC with bone metastasis according to KRAS mutation status. CRC colorectal cancer, $C T x$ chemotherapy, NLR neutrophil-lymphocyte ratio, SRC signet ring cell carcinoma, $A L P$ alkaline phosphatase, CEA carcinoembryonic antigen, No number. ${ }^{a}$ Other bone include skull, rib, scapula, knee, clavicle, sternum. ${ }^{\mathrm{b}}$ Others include fluoropyrimidine based chemotherapy, target monotherapy (cetuximab, bevacizumab, regorafenib).

\section{Results}

Patient characteristics. Of 11,551 CRC patients, colon cancer $(7242,62.7 \%)$ and rectal cancer (4309, $37.3 \%)$ with bone metastasis were diagnosed in 192 (2.7\%) patients and 129 (3.0\%) patients, respectively. Patient characteristics are shown in Table 1. Their median age was 61 years old. There were 188 (58.6\%) male patients. Colon cancer was slightly predominant $(59.8 \%)$. Similar metastatic pattern were observed in both the synchro- 


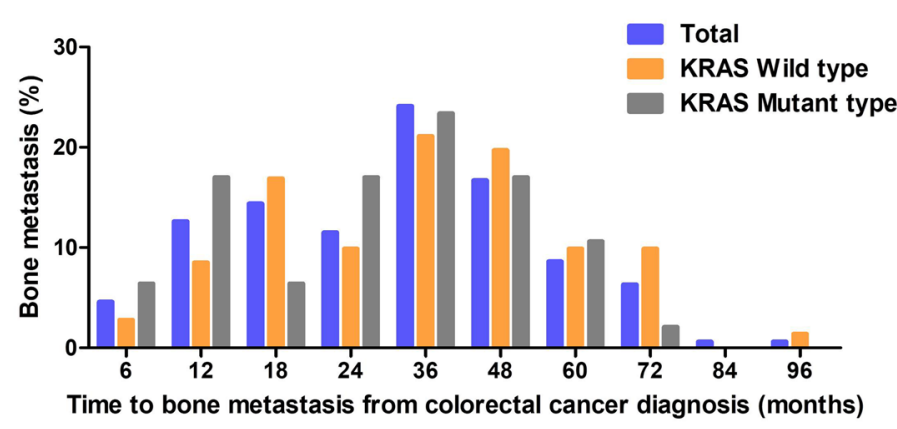

Figure 1. Time to bone metastasis from colorectal cancer (CRC) diagnosis according to KRAS mutation status.

\begin{tabular}{|c|c|c|c|c|c|}
\hline Categorical & No. of patients & Median time (months) & \multicolumn{2}{|c|}{$95 \% \mathrm{CI}$} & P-value \\
\hline \multicolumn{6}{|l|}{ Age } \\
\hline$<61$ & 92 & 27.4 & 24.5 & 30.3 & 0.171 \\
\hline$\geq 61$ & 82 & 25.7 & 20.7 & 30.7 & \\
\hline \multicolumn{6}{|l|}{ Sex } \\
\hline Male & 106 & 27.3 & 23.9 & 30.7 & 0.831 \\
\hline Female & 68 & 25.8 & 20.2 & 31.4 & \\
\hline \multicolumn{6}{|l|}{ Primary site } \\
\hline Colon & 91 & 25.9 & 21.9 & 29.9 & 0.498 \\
\hline Rectum & 83 & 28.3 & 25 & 31.6 & \\
\hline \multicolumn{6}{|l|}{ KRAS mutation } \\
\hline Wild type & 71 & 29 & 23.5 & 34.5 & 0.068 \\
\hline Mutant type & 47 & 25.8 & 15.9 & 35.7 & \\
\hline \multicolumn{6}{|l|}{ Tumor sideness } \\
\hline Left side & 143 & 28.3 & 25.5 & 31.1 & 0.457 \\
\hline Right side & 29 & 22.6 & 14.0 & 31.2 & \\
\hline \multicolumn{6}{|l|}{ Histologic type } \\
\hline Adenocarcinoma or SRC & 164 & 27.4 & 24.8 & 30.0 & 0.269 \\
\hline Mucinous carcinoma & 10 & 16.2 & 11.1 & 21.3 & \\
\hline \multicolumn{6}{|l|}{ Initial clinical stage } \\
\hline 1 or 2 & 19 & 35.9 & 23.2 & 48.6 & $<0.001$ \\
\hline 3 & 68 & 28.8 & 24.1 & 33.5 & \\
\hline 4 & 82 & 20.9 & 16.3 & 25.5 & \\
\hline Overall & 174 & 27.2 & 24.2 & 30.2 & \\
\hline
\end{tabular}

Table 2. Median time from primary cancer diagnosis to bone metastasis in metachronous patients. SRC signet ring cell carcinoma, No number, $C I$ confidence interval.

nous and metachronous groups. There were 292 (91\%) cases of adenocarcinoma. A total of 229 (71.3\%) patients were classified as stage 4 at the initial time of CRC diagnosis. Bone-only metastasis occurred in $28(8.7 \%)$ patients. Frequently observed metastatic sites of bone included the spine (68.8\%) and pelvis $(51.7 \%)$. Median laboratory values of neutrophil-to-lymphocyte ratio (NLR), platelet count, alkaline phosphatase (ALP), and CEA at the time of bone metastasis were $3.5,247 \times 10^{3} / \mu \mathrm{L}, 114 \mathrm{IU} / \mathrm{L}$, and $75.4 \mathrm{ng} / \mathrm{mL}$, respectively. Of patients whose KRAS mutation status was revealed, $126(61.8 \%)$ patients had wild-type KRAS, while $78(38.2 \%)$ patients had KRAS mutations. According to the presence of KRAS mutation, old age, females, and right-sided colon cancer patients were statistically predominant in the group with KRAS mutation. In addition, liver metastasis, lung metastasis, high ALP level and high CEA level were frequently observed at the time of bone metastasis in the group with KRAS mutation. Codon 12 mutation, codon 13 mutation, and mutation site unknown accounted for 58, 16, and 4 cases, respectively. Clinical differences by KRAS mutation type and tumor sidedness were also analyzed in Supplementary Tables S1 and S2. A total of $212(66 \%)$ patients received palliative chemotherapy.

Natural course of bone metastasis. Metachronous bone metastasis occurred in $174(54.2 \%)$ patients (Fig. 1). In metachronous bone metastasis patients, the median TTB was 27.2 months. TTB was analyzed by clinical factors (Table 2). The median TTB in patients with initial clinical stages $1 / 2,3$, and 4 were $35.9,28.8$, and 20.9 months, respectively $(\mathrm{P}<0.001)$. KRAS mutation status was a marginally significant factor affecting time 


\begin{tabular}{|c|c|c|c|c|c|c|c|}
\hline \multirow[b]{2}{*}{ Variable } & \multicolumn{2}{|c|}{$\begin{array}{l}\text { All, No. } \\
\text { (321) }\end{array}$} & \multicolumn{2}{|c|}{$\begin{array}{l}\text { KRAS wild } \\
\text { type, No. } \\
\text { (126) }\end{array}$} & \multicolumn{2}{|c|}{$\begin{array}{l}\text { KRAS } \\
\text { mutant } \\
\text { type, No. } \\
(78)\end{array}$} & \multirow[b]{2}{*}{ P-valu } \\
\hline & No & $\%$ & No & $\%$ & No & $\%$ & \\
\hline SRE, total & 200 & 62.3 & 81 & 64.3 & 55 & 70.5 & 0.359 \\
\hline Pathologic fracture & 26 & 8.1 & 12 & 9.5 & 6 & 7.7 & 0.654 \\
\hline Spinal cord compression & 28 & 8.7 & 13 & 10.3 & 8 & 10.3 & 0.989 \\
\hline Surgery to bone & 43 & 13.4 & 19 & 15.1 & 12 & 15.4 & 0.953 \\
\hline Radiotherapy to bone & 183 & 57 & 75 & 59.5 & 51 & 65.4 & 0.403 \\
\hline Hypercalcemia & 12 & 3.7 & 5 & 4 & 6 & 7.7 & 0.34 \\
\hline
\end{tabular}

Table 3. Skeletal related events. SRE skeletal related event, No number.

to bone metastasis. Patients with KRAS wild-type or mutation had a median TTB of 29 months or 25.8 months, respectively $(\mathrm{P}=0.068)$. In addition, mucinous carcinoma had a shorter tendency of time to bone metastasis compared with that of non-mucinous carcinoma (median TTB, 16.2 months vs. 27.4 months, $\mathrm{P}=0.269$ ). SREs, pathological fractures, spinal cord compression, surgery to bone, radiotherapy to bone, and hypercalcemia were observed in $26(8.1 \%), 28(87 \%), 43(13.4 \%), 183(57 \%)$, and 12 (3.7\%) patients, respectively (Table 3). There was no significant difference in the SRE pattern between patients with KRAS wild-type and patients with KRAS mutation.

Survival analysis. The median follow-up time was 7.4 months. The median OS from diagnosis of bone metastasis was 8.0 months (95\% confidence interval (CI) 6.8-9.2 months). The statistically determined best cut-off point of NLR was 2.7. Survival was significantly extended in patients with low levels of NLR or ALP compared to patients with high levels of NLR or ALP (median OS, NLR: 12.2 months vs. 5.6 months, $\mathrm{P}<0.001$; ALP: 11.2 months vs. 5.2 months, $\mathrm{P}<0.001$; Fig. $2 \mathrm{~A}, \mathrm{~B}$ ). Patients with bone-only metastasis had the longest median OS of 20.4 months (Fig. 2C). Peritoneal carcinomatosis showed poor survival outcome (median OS: 9.2 months vs. 4.3 months, $\mathrm{P}<0.001$ ) (Fig. 2D). However, KRAS mutation status did not significantly affect survival outcome (median OS: wild-type vs. mutant type, 9.2 months vs. 8.4 months, $\mathrm{P}=0.083$ ).

According to univariate analysis, primary site; tumor sidedness; bone metastasis pattern (bone-only or multiorgan metastasis); presence of liver, lung or peritoneal metastasis; NLR; ALP level; and CEA level were all associated with $O S$ at $\mathrm{p}$-value $<0.05$ (Table 4). Multivariate analysis demonstrated that multi-organ metastasis (Hazard ratio (HR) 2.43; 95\% CI 1.39-4.25), peritoneal metastasis (HR 1.47; 95\% CI 1.08-2.02), NLR $\geq 2.7$ (HR 1.54; 95\% CI 1.17-2.04), and ALP $\geq 123$ (HR 1.76; 95\% CI 1.32-2.34) were independent factors for OS. Bone-only metastasis was a prognostic factor with the highest hazard ratio for OS.

Prognostic model analysis. We divided patients into two subgroups based on four independent prognostic factors for OS: 171 patients with 0 to 2 adverse prognostic factors (low-risk group) and 122 patients with three to four adverse prognostic factors (high-risk group). These risk groups were significantly associated with OS (median OS for low- and high-risk groups: 11.9 months and 4.1 months, respectively, $\mathrm{P}<0.001$ ) (Fig. 3A). Regardless of the risk group, palliative chemotherapy had a benefit for OS in patients with bone metastasis. Among 171 low-risk patients, 123 (71.9\%) patients received palliative chemotherapy. As shown in Fig. 3B, patients treated with palliative chemotherapy had longer OS than patients who received the best supportive care, including radiotherapy (median OS: 16.7 months vs. 4.3 months respectively, $\mathrm{P}<0.001$ ). Among 122 highrisk patients, OS was also longer in the palliative chemotherapy group (median OS: 6.8 months vs. 2.3 months, $\mathrm{P}<0.001$ ) (Fig. 3C).

\section{Discussion}

Bone metastasis of CRC is rare, but it is related to SREs. Most patients have other organ metastasis and survival is 8.0 months in unselected patients. KRAS mutation affected different clinical manifestations in these patients. We showed that patients with KRAS mutation had a tendency to have shorter time to bone metastasis than patients with wild-type KRAS, but there were no significant survival differences between patients with KRAS wild-type and those with KRAS mutation. Several prognostic factors were noted in this study. Patients who received palliative chemotherapy showed prolonged survival compared to patients with supportive care, especially in patients with less adverse factors.

The observed frequency of bone metastasis in CRC was $1.2-12 \%$, with a wide range ${ }^{3,4}$. Each study included $\mathrm{CRC}$ with bone metastasis in various clinical settings dealing with resected patients or metastatic patients. It might be related to the wide range of bone metastasis incidence in CRC patients. Recently, systemic chemotherapy was more effective and survival was prolonged. In addition, diagnostic modality such as positron emission tomography (PET) coupled with computed tomography (CT) scan (PET-CT) was developed and possibility of bone metastasis detection would be increased. Asymptomatic bone metastasis could be detected on routine follow-up. It might help prevent SREs, but it has a possibility of overtreatment, such as chemotherapy regimen change or radiotherapy. Therefore, the role of asymptomatic bone metastasis detection should be investigated in 
A

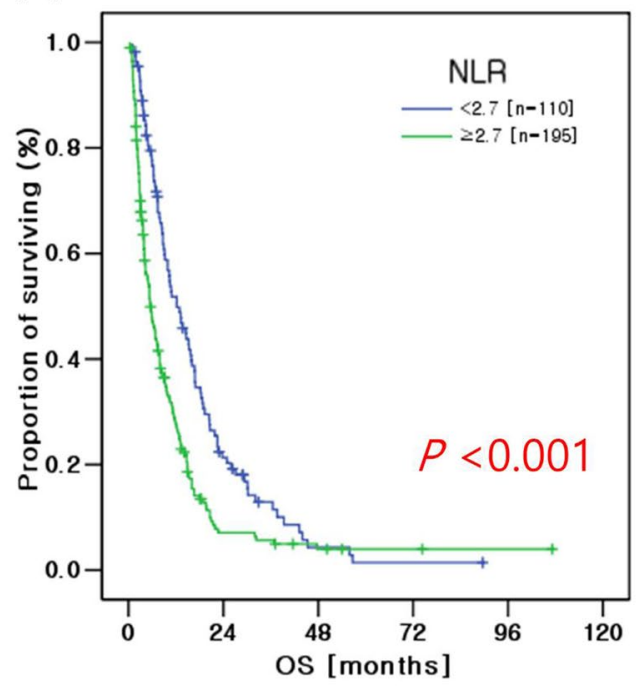

C

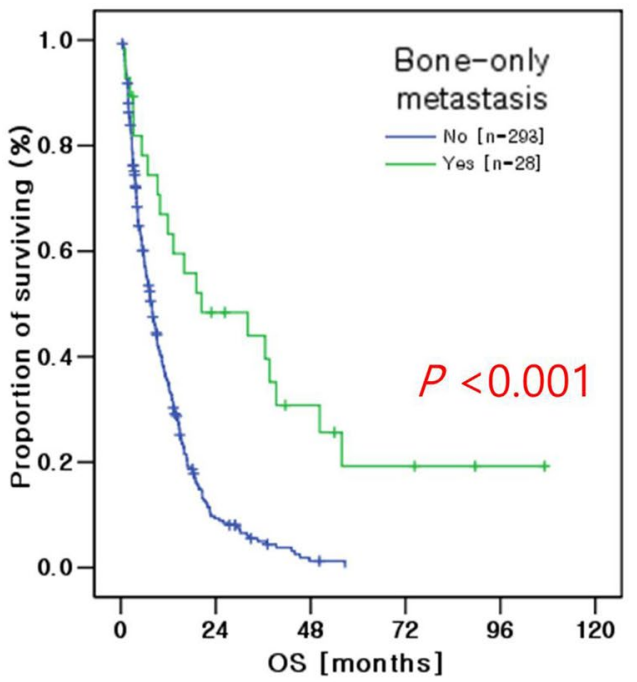

B

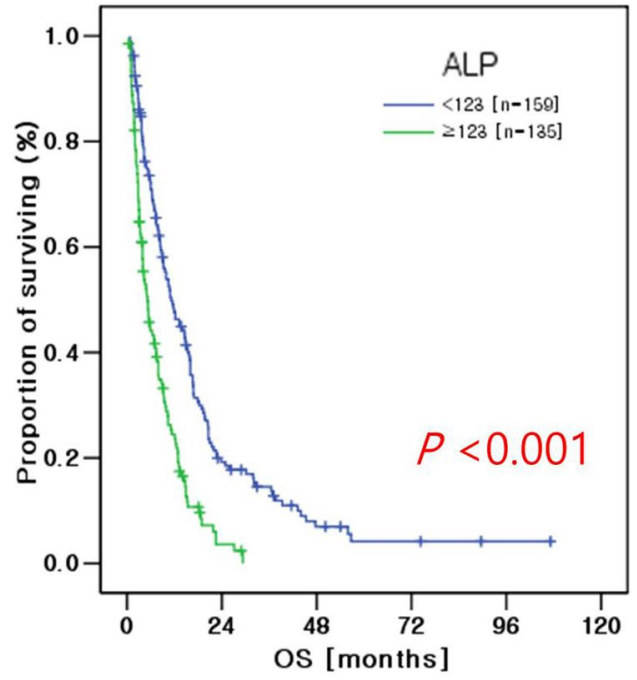

D

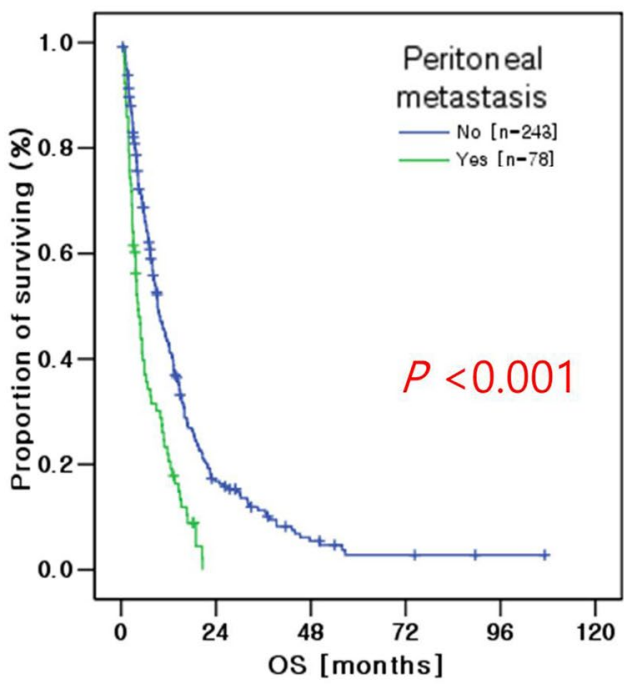

Figure 2. Kaplan-Meier curves of overall survival (OS) for all patients $(n=321)$ according to prognostic factors: (A) neutrophil-to-lymphocyte ratio (NLR), (B) alkaline phosphatase (ALP), (C) bone-only metastasis, and (D) peritoneal metastasis.

further studies. In contrast, surveillance cannot be performed until death in various circumstances, and routine follow-up schemes without bone scintigraphy or PET-CT may not detect bone metastasis. These factors are related to the underdiagnosis of bone metastasis.

In clinical practice, at the time of bone metastasis, multi-organ metastases were frequently observed. Boneonly metastasis was found in only $8.7 \%$ of subjects, and $91.3 \%$ of patients had other organ metastasis in this study. Commonly observed multi-organ metastases might be related to the poor prognosis in bone metastasis ${ }^{5}$. KRAS mutation has been studied as a metastasis promoting factor in CRC. The results of our study also showed that patients with KRAS mutation had a higher frequency of both lung and liver metastases at the time of bone metastasis. In addition, patients with KRAS mutation more frequently had high ALP and high CEA levels. Regarding KRAS mutation type (codon 12 vs. codon 13), there were no significant clinical differences between these two groups, except the NLR level.

The median time to bone metastasis was 27.2 months in metachronous patients. Time to bone metastasis was shorter in patients with KRAS mutation compared to patients with wild type KRAS, although it was not statistically significant. It has been reported that KRAS mutation can lead to shorter time to metastasis in liver, lung, and bone ${ }^{17}$. Initial CRC stage was also a significant factor for time to bone metastasis. Based on our data, first surveillance for identification of bone metastasis might be considered within 2 years after primary CRC diagnosis and attention is needed, especially in patients with initial stage 4 . Additional evaluation and close monitoring 2 years after CRC diagnosis should also be performed.

Risk factors for bone metastasis in CRC have been reported in many studies ${ }^{8}$. Primary tumor location, tumor stage and histologic type were revealed as significant risk factors for bone metastasis. Regarding tumor location, 


\begin{tabular}{|c|c|c|c|c|c|c|c|c|c|}
\hline \multirow[b]{2}{*}{ Categorical } & \multicolumn{5}{|l|}{ Univariate } & \multicolumn{4}{|c|}{ Multivariate } \\
\hline & No. of patients & HR & \multicolumn{2}{|c|}{ 95\% CI } & P-value & HR & \multicolumn{2}{|c|}{$95 \% \mathrm{CI}$} & P-value \\
\hline \multicolumn{10}{|l|}{ Age } \\
\hline$<61$ & 154 & 1 & & & 0.057 & & & & \\
\hline$\geq 61$ & 167 & 1.26 & 0.99 & 1.59 & & & & & \\
\hline \multicolumn{10}{|l|}{ Sex } \\
\hline Male & 188 & 1 & & & 0.876 & & & & \\
\hline Female & 133 & 0.98 & 0.77 & 1.25 & & & & & \\
\hline \multicolumn{10}{|l|}{ Primary site } \\
\hline Colon & 192 & 1 & & & 0.041 & & & & \\
\hline Rectum & 129 & 0.78 & 0.61 & 0.99 & & & & & \\
\hline \multicolumn{10}{|l|}{ KRAS mutation } \\
\hline Wild type & 126 & 1 & & & 0.085 & & & & \\
\hline Mutant type & 78 & 1.31 & 0.96 & 1.79 & & & & & \\
\hline \multicolumn{10}{|l|}{ Tumor sideness } \\
\hline Left side & 250 & 1 & & & 0.018 & & & & \\
\hline Right side & 63 & 1.44 & 1.06 & 1.94 & & & & & \\
\hline \multicolumn{10}{|l|}{ Metastasis pattern } \\
\hline Synchronous & 147 & 1 & & & 0.815 & & & & \\
\hline Metachronous & 174 & 0.97 & 0.77 & 1.23 & & & & & \\
\hline \multicolumn{10}{|l|}{ Histologic type } \\
\hline Adenocarcinoma & 292 & 1 & & & 0.727 & & & & \\
\hline SRC & 14 & 1.15 & 0.66 & 2.00 & & & & & \\
\hline Mucinous carcinoma & 15 & 0.84 & 0.48 & 1.47 & & & & & \\
\hline \multicolumn{10}{|l|}{ Bone-only metastasis } \\
\hline Yes & 28 & 1 & & & $<0.001$ & 1 & & & 0.002 \\
\hline No & 293 & 3.02 & 1.88 & 4.87 & & 2.43 & 1.39 & 4.25 & \\
\hline \multicolumn{10}{|l|}{ Liver metastasis } \\
\hline No & 137 & 1 & & & $<0.001$ & & & & \\
\hline Yes & 184 & 1.87 & 1.45 & 2.40 & & & & & \\
\hline \multicolumn{10}{|l|}{ Lung metastasis } \\
\hline No & 153 & 1 & & & 0.022 & & & & \\
\hline Yes & 168 & 1.32 & 1.04 & 1.67 & & & & & \\
\hline \multicolumn{10}{|l|}{ Peritoneal metastasis } \\
\hline No & 243 & 1 & & & $<0.001$ & 1 & & & 0.016 \\
\hline Yes & 78 & 2.05 & 1.56 & 2.71 & & 1.47 & 1.08 & 2.02 & \\
\hline \multicolumn{10}{|l|}{ Brain metastasis } \\
\hline No & 306 & 1 & & & 0.172 & & & & \\
\hline Yes & 15 & 1.46 & 0.85 & 2.50 & & & & & \\
\hline \multicolumn{10}{|l|}{ NLR } \\
\hline$<2.7$ & 110 & 1 & & & $<0.001$ & 1 & & & 0.002 \\
\hline$\geq 2.7$ & 195 & 1.72 & 1.33 & 2.21 & & 1.54 & 1.17 & 2.04 & \\
\hline \multicolumn{10}{|l|}{$\operatorname{ALP}(I U / L)$} \\
\hline$<123$ & 159 & 1 & & & $<0.001$ & 1 & & & $<0.001$ \\
\hline$\geq 123$ & 135 & 2.17 & 1.67 & 2.81 & & 1.76 & 1.32 & 2.34 & \\
\hline CEA $(\mathrm{ng} / \mathrm{mL})$ & & & & & & & & & \\
\hline$<5$ & 37 & 1 & & & $<0.001$ & & & & \\
\hline$\geq 5$ & 253 & 2.29 & 1.52 & 3.45 & & & & & \\
\hline Palliative CTx & & & & & & & & & \\
\hline No & 109 & 1 & & & $<0.001$ & & & & \\
\hline Yes & 212 & 0.25 & 0.20 & 0.33 & & & & & \\
\hline
\end{tabular}

Table 4. Univariate and multivariate analysis using cox regression for OS. NLR neutrophil-lymphocyte ratio, $A L P$ alkaline phosphatase, $C E A$ carcinoembryonic antigen, $C T x$ chemotherapy, $S R C$ signet ring cell carcinoma, $O S$ overall survival, No number, $H R$ hazard ratio, $C I$ confidence interval. 
A

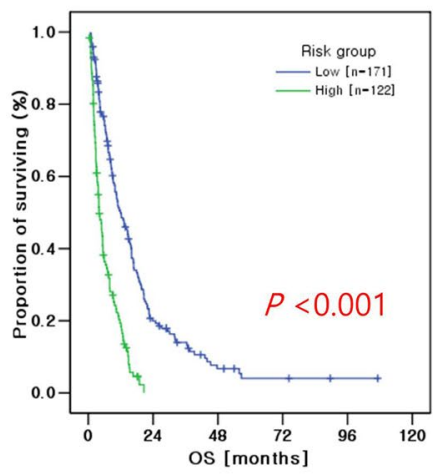

B

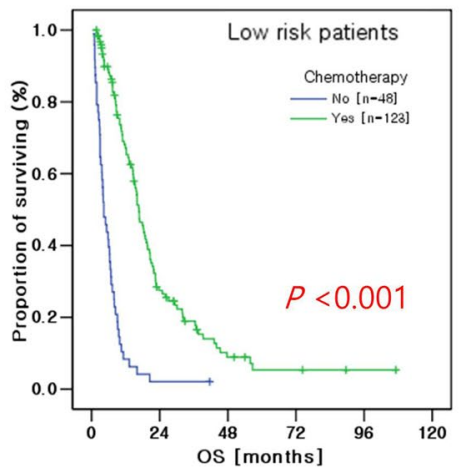

C

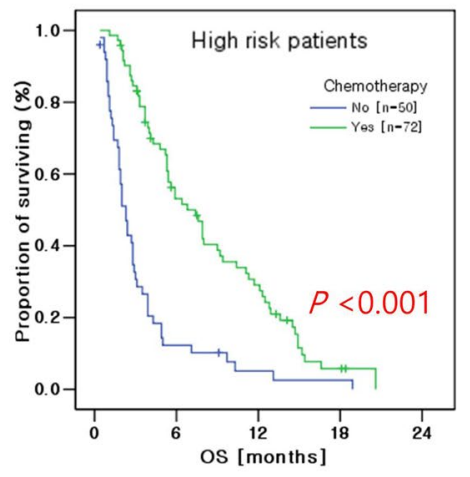

Figure 3. Kaplan-Meier overall survival (OS) curves according to risk group defined by number of adverse prognostic factors: (A) low-risk ( 0 to 2 adverse factors) and high-risk ( 3 to 4 adverse factors) groups, (B) palliative chemotherapy in the low-risk patients, and (C) palliative chemotherapy in the high-risk patients.

other studies have shown that rectal cancer had a greater chance of bone metastasis than colon cancer. Although our studies showed more of a tendency for bone metastasis in rectal cancer compared to that in colon cancer, there was no significant difference between the two groups $(\mathrm{P}=0.28)$. This study included all CRC patients treated in our institution, and the distribution of clinical factors, such as stage and histology, may have affected the results in the two groups.

The prognosis of bone metastasis in CRC patients was poor. The median survival of these patients was approximately 8 months ${ }^{7,18}$. Prognostic factors for bone metastasis in CRC have been evaluated in several studies. Multiple bone metastases, primary site of colon/lung metastasis, and elevated CEA level have been identified as poor prognostic factors. In our study, four prognostic factors were revealed: multi-organ metastasis, peritoneal metastasis, high NLR ( $\geq 2.7)$, and high ALP ( $\geq 123 \mathrm{IU} / \mathrm{L})$. Of these factors, whether a patient had bone-only metastasis was a prognostic factor with the highest hazard ratio for OS (HR 2.43; 95\% CI 1.39-4.25). The median survival was 7.8 months for patients with other organ metastasis and 20.4 months for those with bone-only metastasis. We also made a prognosis scoring model using the four independent factors. Risk group was categorized as low-risk (score 0-2) and high-risk (score 3-4) groups. The median survival of the low-risk group was 11.9 months. It was almost 1.5-fold (4 months longer) than that of unselected CRC patients with bone metastasis.

Bone metastasis is an important factor for quality of life and survival. Proper treatment, including radiotherapy, surgery and medical therapy, improves bone metastasis symptoms, such as pain, and bisphosphonates could delay the development of SREs ${ }^{8}$. In addition, bone metastasis has been known to be a poor prognostic factor in metastatic CRC. In our study, palliative chemotherapy provided patients a chance to increase survival up to 16.7 months in the low-risk group. The high-risk group also had a survival gain from palliative chemotherapy, although the survival benefit was less than the low-risk group. We had to pay attention to the interpretation of this survival data analyzed by the presence of chemotherapy, because patients who did not receive chemotherapy might have poor performance status or comorbidities, which might be related to poor prognosis. Our results suggest the possibility of chemotherapy benefit in bone metastasis patients, and predictive markers for chemotherapy should be further studied. A prognosis scoring model might help predict the survival for CRC patients with bone metastasis and guide treatment decisions, including palliative chemotherapy, or the best supportive care according to their benefits.

This study had several limitations. First, it was a retrospective study. Thus, the performance status could not be identified. Second, this study did not evaluate the impact of bisphosphonates on SREs. Bisphosphonates are not reimbursed in Korea. They are only permitted in hypercalcemia secondary to CRC. However, this study also has its strengths. Large numbers of patients were enrolled, and KRAS mutation status and tumor sidedness were analyzed to identify their roles in bone metastasis of CRC. In conclusion, bone metastasis of CRC is rare, but it is related to SREs. Most patients have other organ metastasis and survival is 8.0 months in unselected patients. It should receive more attention from clinicians. Time to bone metastasis and prognosis were not different according to KRAS mutation status in our study. Surveillance for bone metastasis might be considered in the first 2 years after CRC diagnosis, and additional evaluation and close monitoring 2 years after the CRC diagnosis should be performed. According to our prognosis scoring model, a patient's prognosis could be predicted, and it may help to guide palliative chemotherapy in CRC with bone metastasis.

\section{Methods}

Patient selection. From January 2006 to April 2016, a total of 11,551 patients were diagnosed with CRC at Severance Hospital, Seoul, Korea. Diagnosis was made from surgical excision or tissue biopsy. Inclusion criteria were as follows: (1) age > 18 years, (2) histologically confirmed diagnosis of CRC, (3) radiologically or pathologically confirmed bone metastasis, and (4) available electronic medical records (including treatment information). Patients with other histology types, such as neuroendocrine tumors, small cell carcinoma, melanoma, or lymphoma, were excluded. A total of 321 patients fulfilled the inclusion and exclusion criteria. The Severance Hospital Institutional Review Board (IRB) approved this retrospective study, and the requirement to obtain 
informed consent was waived. This study was conducted in accordance with the Declaration of Helsinki and was consistent with Good Clinical Practice.

Data collection. To diagnose bone metastasis, plain radiography, technetium-99m methylene diphosphonate (Tc-99m MDP) whole body bone scintigraphy, CT scan, PET-CT and magnetic resonance imaging (MRI) were performed. All available correlative radiographic studies were reviewed. The following baseline data were recorded at the time of bone metastasis: age, sex, tumor location, histologic type, initial stage, metastatic organ, and KRAS mutation status. For tumor sidedness determination, the splenic flexure was used for differentiation of the left side of the colon and right side of the colon. KRAS mutation status was identified by Sanger sequencing, the peptide nucleic acid (PNA)-mediated PCR clamping method ${ }^{19}$, or pyrosequencing. Sanger sequencing and the PNA-mediated PCR clamping method were used to detect mutations in codons 12 and 13 . Pyrosequencing was performed to detect mutations at codons 12,13, and 61. Metachronous bone metastasis was defined as the time to bone metastasis being greater than 3 months from initial CRC diagnosis.

Hematological and blood chemistry values included NLR, platelet count, ALP, and CEA. The NLR was calculated by dividing the absolute neutrophil count by the absolute lymphocyte count. Parameters related to bone metastasis included time to bone metastasis, site of bone metastasis, and SREs defined by pathologic fracture, spinal cord compression, surgery to bone, radiotherapy to bone, and hypercalcemia. After the diagnosis of bone metastasis, whether patients received palliative chemotherapy was also reviewed.

Statistical analysis. The demographic and clinical characteristics were compared using the Chi square or Fisher exact test. The Mann-Whitney U test was used for comparisons of continuous variables. In patients with metachronous bone metastasis, freedom from bone metastasis since CRC diagnosis was estimated using the Kaplan-Meier method. Overall survival (OS) was defined as the time from the date of bone metastasis diagnosis to death from any cause. Hematological and blood chemistry values were initially recorded as continuous variables and later transformed into categorical variables according to lower or upper normal values (platelet count, ALP level, and CEA level) or the best cut-off point (NLR) determined by the Contal and O'Quigley method, which calculates the maximization of the hazard ratio based on log rank statistics and estimates the best cut-off value $^{20}$. Survival curves were generated using the Kaplan-Meier method and compared using the log-rank test.

Univariate analysis was performed to determine associations of OS with the following prognostic factors: age; sex; tumor location and sidedness; timing of bone metastasis (synchronous or metachronous); histologic type; bone metastasis pattern (bone-only or not); liver, lung, peritoneal, and/or brain metastasis; NLR; ALP level; CEA level; KRAS mutation status; and palliative chemotherapy treatment. A stepwise multivariate analysis with Cox proportional hazard model was performed using significant factors from the univariate analysis. Hazard ratio (HR), 95\% CI, and $\chi^{2}$ scores were obtained for all regressions. Statistical analyses were performed using PASW Statistics 18.0 (SPSS Inc., Chicago, IL, USA), SAS version 9.2 (SAS institute, Cary, NC, USA), and R version 3.1.3 (Institute for Statistics and Mathematics, Vienna, Austria www.R-project.org).

\section{Data availability}

Upon reasonable request, data and material are available from the corresponding authors.

Received: 18 April 2020; Accepted: 11 November 2020

Published online: 03 December 2020

\section{References}

1. Siegel, R. L., Miller, K. D. \& Jemal, A. Cancer statistics, 2019. CA Cancer J. Clin. 69, 7-34. https://doi.org/10.3322/caac.21551 (2019).

2. Riihimaki, M., Hemminki, A., Sundquist, J. \& Hemminki, K. Patterns of metastasis in colon and rectal cancer. Sci. Rep. 6, 29765. https://doi.org/10.1038/srep29765 (2016).

3. Hernandez, R. K. et al. Incidence of bone metastases in patients with solid tumors: analysis of oncology electronic medical records in the United States. BMC Cancer 18, 44. https://doi.org/10.1186/s12885-017-3922-0 (2018).

4. Nozue, M. et al. Treatment and prognosis in colorectal cancer patients with bone metastasis. Oncol. Rep. 9, 109-112 (2002).

5. Liu, F. et al. Prognostic risk factors in patients with bone metastasis from colorectal cancer. Tumour Biol. https://doi.org/10.1007/ s13277-016-5465-4 (2016).

6. Prasanna, T. et al. The survival outcome of patients with metastatic colorectal cancer based on the site of metastases and the impact of molecular markers and site of primary cancer on metastatic pattern. Acta Oncol. 57, 1438-1444. https://doi.org/10.1080/02841 86X.2018.1487581 (2018).

7. Santini, D. et al. Natural history of bone metastasis in colorectal cancer: final results of a large Italian bone metastases study. Ann. Oncol. 23, 2072-2077. https://doi.org/10.1093/annonc/mdr572 (2012).

8. Christensen, T. D., Jensen, S. G., Larsen, F. O. \& Nielsen, D. L. Systematic review: incidence, risk factors, survival and treatment of bone metastases from colorectal cancer. J. Bone Oncol. 13, 97-105. https://doi.org/10.1016/j.jbo.2018.09.009 (2018).

9. Vaughn, C. P., Zobell, S. D., Furtado, L. V., Baker, C. L. \& Samowitz, W. S. Frequency of KRAS, BRAF, and NRAS mutations in colorectal cancer. Genes Chromosomes Cancer 50, 307-312. https://doi.org/10.1002/gcc.20854 (2011).

10. Tan, C. \& Du, X. KRAS mutation testing in metastatic colorectal cancer. World J. Gastroenterol. 18, 5171-5180. https://doi. org/10.3748/wjg.v18.i37.5171 (2012).

11. Kemeny, N. E. et al. KRAS mutation influences recurrence patterns in patients undergoing hepatic resection of colorectal metastases. Cancer 120, 3965-3971. https://doi.org/10.1002/cncr.28954 (2014).

12. Hutchins, G. et al. Value of mismatch repair, KRAS, and BRAF mutations in predicting recurrence and benefits from chemotherapy in colorectal cancer. J. Clin. Oncol. 29, 1261-1270. https://doi.org/10.1200/JCO.2010.30.1366 (2011).

13. Pereira, A. A. et al. Association between KRAS mutation and lung metastasis in advanced colorectal cancer. Br. J. Cancer 112, 424-428. https://doi.org/10.1038/bjc.2014.619 (2015).

14. Phipps, A. I. et al. KRAS-mutation status in relation to colorectal cancer survival: the joint impact of correlated tumour markers. Br. J. Cancer 108, 1757-1764. https://doi.org/10.1038/bjc.2013.118 (2013). 
15. Roth, A. D. et al. Prognostic role of KRAS and BRAF in stage II and III resected colon cancer: results of the translational study on the PETACC-3, EORTC 40993, SAKK 60-00 trial. J. Clin. Oncol. 28, 466-474. https://doi.org/10.1200/JCO.2009.23.3452 (2010).

16. Guo, F. et al. Mutation status and prognostic values of KRAS, NRAS, BRAF and PIK3CA in 353 Chinese colorectal cancer patients. Sci. Rep. 8, 6076. https://doi.org/10.1038/s41598-018-24306-1 (2018).

17. Yaeger, R. et al. RAS mutations affect pattern of metastatic spread and increase propensity for brain metastasis in colorectal cancer. Cancer 121, 1195-1203. https://doi.org/10.1002/cncr.29196 (2015).

18. Portales, F. et al. Bone metastases in gastrointestinal cancer. Clin. Exp. Metast. 32, 7-14. https://doi.org/10.1007/s10585-014-9686-x (2015).

19. Kwon, M. J., Lee, S. E., Kang, S. Y. \& Choi, Y. L. Frequency of KRAS, BRAF, and PIK3CA mutations in advanced colorectal cancers: comparison of peptide nucleic acid-mediated PCR clamping and direct sequencing in formalin-fixed, paraffin-embedded tissue. Pathol. Res. Pract. 207, 762-768. https://doi.org/10.1016/j.prp.2011.10.002 (2011).

20. Contal, C. O. Q. J. An application of changepoint methods in studying the effect of age on survival in breast cancer. Comput. Stat. Data Anal. 30, 253-270 (1999).

\section{Acknowledgements}

This research was supported by Basic Science Research Program through the National Research Foundation of Korea (NRF) funded by the Ministry of Education (2020R1F1A1066973).

\section{Author contributions}

Conception/design: H.S.P. and J.B.A. Acquisition of data: Y.J.C., H.S.K., J.H.K., C.L., S.-H.B., S.J.S. and J.B.A. Analysis and interpretation: H.S.P. and J.B.A. Manuscript writing: H.S.P. and J.B.A. Final approval of manuscript: H.S.P., Y.J.C., H.S.K., J.H.K., C.L., S.-H.B., S.J.S. and J.B.A.

\section{Competing interests}

The authors declare no competing interests.

\section{Additional information}

Supplementary Information The online version contains supplementary material available at https://doi. org/10.1038/s41598-020-78253-x.

Correspondence and requests for materials should be addressed to J.B.A.

Reprints and permissions information is available at www.nature.com/reprints.

Publisher's note Springer Nature remains neutral with regard to jurisdictional claims in published maps and institutional affiliations.

Open Access This article is licensed under a Creative Commons Attribution 4.0 International License, which permits use, sharing, adaptation, distribution and reproduction in any medium or format, as long as you give appropriate credit to the original author(s) and the source, provide a link to the Creative Commons licence, and indicate if changes were made. The images or other third party material in this article are included in the article's Creative Commons licence, unless indicated otherwise in a credit line to the material. If material is not included in the article's Creative Commons licence and your intended use is not permitted by statutory regulation or exceeds the permitted use, you will need to obtain permission directly from the copyright holder. To view a copy of this licence, visit http://creativecommons.org/licenses/by/4.0/.

(C) The Author(s) 2020 